\title{
Percepción de los estudiantes sobre la utilización de screencast en la enseñanza de la física
}

J.A. Gómez-Tejedor, A. Vidaurre, J. Riera, J.M. Meseguer-Dueñas, J. Molina-Mateo

ETS de Ingeniería del Diseño, Universitat Politècnica de València (España)

\begin{abstract}
In recent years, screencast has been widely used as a teaching tool in different areas. A screencast is a short video obtained from the capture of the computer screen with narrator explanation provided by the teacher. This paper analyse the opinion of Freshmen University in relation to the benefits that the use of screencast contributes to improve learning of physics, how the students use the screencast, and also why they choose to use it or do not. Several screencasts have been prepared and uploaded to the Sakai/PoliformaT platform in which key and difficult points of physics are addressed. Sakai/PoliformaT is a learning platform developed at the Universitat Politècnica de València (UPV, www.upv.es/index-en.htm), through the Sakai project (https://sakaiproject.org). On average, each screencast was watched more than 100 times from a group of physics with 117 students enrolled. Previous works suggested that screencast is perceived by students as beneficial and concluded that learning improvements are obtained. To verify this perception, an anonymous survey, consisting of 10 multiple choice questions and an open question was done. The relationship between the use of the screencast and the perception students have about the improvement of understanding of the subject is analyzed. The relationship between the use of screencast and results in certain exam questions are also discussed. The results indicate that most students believe that the screencast are useful for learning by helping them to understand relevant concepts and thus improving their results.
\end{abstract}

Keywords: Screencast, physics

\footnotetext{
Resumen

En los últimos años, screencast se ha utilizado ampliamente como herramienta de enseñanza en diferentes áreas. Un screencast es un video corto obtenido a partir de la captura de la pantalla del ordenador acompañada de la narración de un profesor. Este trabajo explora la opinión de los estudiantes de primer curso de Universidad en relación con los beneficios que el uso de screencast aporta a la mejora del aprendizaje de la física, con el uso que los estudiantes hacen del screencast, y también por qué deciden usarlo o no. Se han preparado y subido a la plataforma
} 
Sakai/PoliformaT varios screencasts en los que se tratan aspectos clave de la física en los que es sabido que los estudiantes encuentran dificultades. Sakai/PoliformaT es una plataforma de aprendizaje desarrollada en la Universitat Politècnica de València (UPV, www.upv.es/index-en.htm), a través del proyecto Sakai (https://sakaiproject.org). En promedio, cada screencast fue visionado más de 100 veces, por estudiantes de un grupo de física de 117 alumnos. Trabajos previos sugieren que screencast es percibido por los alumnos como beneficioso y concluyen que se obtienen mejoras en el aprendizaje. Para verificar esta percepción, se realizó una encuesta anónima que constaba de 10 preguntas de respuesta múltiple y una pregunta abierta. Se analizó la relación entre el uso de los screencast y la percepción de los estudiantes de haber obtenido beneficios en cuanto a una mejor comprensión de la materia tratada. También se analizó la relación entre el uso de screencast y la nota en preguntas relacionadas de un examen. Los resultados indican que la mayoría de los estudiantes opinan que los screencast son útiles para su aprendizaje ayudándoles a comprender conceptos relevantes y mejorando así sus resultados.

Palabras clave: Screencast, física.

\section{Introducción}

Un screencast es un video tutorial que mezcla la imagen de un ordenador con la explicación de la misma mediante la de voz de un experto. Screencast combina información visual y de audio y es accesible a través de internet (Peterson 2007). Puede ser visto en cualquier dispositivo con capacidad para visionar vídeos, principalmente un ordenador, aunque también puede ser un teléfono móvil o una tableta. Screencast es una herramienta muy útil para la enseñanza de materias basadas en habilidades, como la física o las matemáticas, porque permite visualizar soluciones de problemas paso a paso, incluyendo notación específica (Jordan et al. 2012). Hoy en día la producción de screencast es sencilla con muchas posibilidades de software de grabación, algunas gratis.

Screencast es una tecnología de enseñanza aprendizaje efectiva en la era digital. Permite aprendizaje personalizado y flexible. Screencasting puede ser considerado como una herramienta de distribución de contenidos y una herramienta de evaluación, bien en enseñanza a distancia o presencial. Puede usarse para mejorar la calidad del aprendizaje en la enseñanza universitaria (Tunku Ahmad y Doheny 2014). En la enseñanza presencial, supone un suplemento a las clases y favorece el aprendizaje de aspectos claves. También ayuda a los estudiantes que no han podido acudir a clase. Screencasts ofrece múltiples beneficios, especialmente en materias que tratan conceptos complejos y de resolución de problemas como ingeniería, matemáticas y física. Estas materias tienden a tener numerosos conceptos que sobrepasan la capacidad de comprender de los estudiantes asistiendo a una sola clase. Habitualmente los tutores ofrecen asistencia personalizada lo que les obliga a una alta dedicación en fechas específicas, como puede ser en la preparación de un examen.

(cc) EY-NC-ND 2016, Universitat Politècnica de València

Congreso IN-RED (2016) 
Por tanto, las Tecnologías de la Información y Comunicación (TIC), a través de los screencasts ayudan a gestionar esta cantidad de trabajo facilitando a los estudiantes a entender estos conceptos difíciles (Merkt et al. 2011). Screencast da a los estudiantes el control sobre la cantidad de información que pueden ver en una sesión; ellos deciden si van hacia adelante, pausan o repiten un fragmento de screencast.

Cinco aproximaciones han sido identificadas para estas herramientas de enseñanza: proporcionar una vista rápida; describir procedimientos; presentar conceptos; elaborar contenidos y focalizar la atención (Sugar, Brown y Luterbach 2010). El diseño pedagógico es muy importante para producir screencast efectivos y se han publicado algunas guías (Oud 2009). Entre ellos, se ha demostrado que los screencast cortos con un único objetivo son mejores que la réplica del contenido disponible en otro medio (Sutton-Brady et al. 2011; Jordan et al. 2012). En resumen, la combinación de gráficas con la narración mejora el proceso de aprendizaje cuando es comparado con el texto en formato tradicional (Mayer 2014).

En este estudio analizamos la opinión de los estudiantes sobre una serie de screencast desarrollados por los profesores de la asignatura de física de primer curso de la Universitat Politècnica de València (UPV, http://www.upv.es) del grado de Ingeniería Aeroespacial. Algunos de los screencast están relacionados con sesiones teóricas y otros muestran, a través de vídeos cortos, cómo resolver problemas típicos que permiten a los estudiantes superar las dificultades.

El objetivo de este trabajo es responder a tres preguntas de investigación acerca del uso de los screencast:

- ¿Los estudiantes incorporan el screencast como parte del material de apoyo al aprendizaje?

- ¿Los estudiantes consideran que el screencast es útil para su estrategia de aprendizaje?

- ¿Prefieren el screencast a otro tipo de materiales de apoyo?

Así mismo, en este trabajo planteamos si existen beneficios académicos para los estudiantes por la utilización de los screencast. Para ello hemos implementado una prueba en donde parcialmente se recogen contenidos abordados por los screencast suministrados. Correlacionando la nota obtenida con la utilización de los screencast, pretendemos realizar una primera aproximación, que nos pueda dar indicios sobre su influencia en el rendimiento académico de los alumnos. De esta manera, planteamos una cuarta pregunta de investigación, cuyo enunciado sería

- ¿Mejoran los screencast el rendimiento académico de los alumnos?

\section{Metodología}

Como se ha dicho, los screencasts se han preparado y utilizado para la materia de física en los cursos universitarios de primer año de carreras de ingeniería. No sólo los temas,

2016, Universitat Politècnica de València

Congreso In-Red (2016) 
contenidos y objetivos de las grabaciones de pantalla eran bastante variados, sino que el formato y aplicación también lo eran, dependiendo de la motivación, la aplicación, el objetivo de los estudiantes y de la clase:

- Conceptos teóricos, ejemplos de resolución de problemas e introducción de prácticas de laboratorio.

- Screencasts, incluyendo la imagen y la voz del profesor o sólo la voz.

- Transparencias, con o sin anotaciones a mano.

\subsection{Encuesta de satisfacción a los estudiantes}

Se llevó a cabo una encuesta anónima para obtener la opinión de los estudiantes sobre los screencast con el propósito de mejorar la metodología. La encuesta fue diseñada analizando la literatura (Vincelette y Bostic 2013; Dunn, McDonald y Loch 2015; McDonald et al. 2013; Green, Pinder-Grover y Millunchick 2012). La encuesta constaba de 11 preguntas (véase la lista en la Tabla 1) y fue respondida por un total de 60 alumnos que asistían a clase con diferentes profesores. Todas las preguntas eran cuantitativas, a excepción de la pregunta 11, que era una pregunta abierta. En las preguntas 2, 3, 4 y 10 los estudiantes debían elegir de una lista de opciones, y las otras preguntas fueron valoradas en una escala Likert de cinco puntos (Likert 1932). En la pregunta 1, la opción "No he visto el screencast" se ha agregado a la escala de cinco puntos. Los porcentajes se han calculado sobre el total de alumnos que han visto los screencast, salvo en las preguntas más generales en las que se considera el número de alumnos que contestan.

La pregunta abierta se analizó utilizando un análisis de contenido temático inductivo (TCA). Las respuestas individuales de los estudiantes fueron leídas, en busca de ideas clave específicas, y luego se clasificaron de acuerdo a la similitud de las ideas clave compartidas entre los estudiantes (Tunku Ahmad y Doheny 2014). El análisis inductivo es un proceso de codificación de los datos sin tratar de encajarlos en un marco de codificación preexistente (Braun y Clarke 2006).

\section{Tabla 1. Encuesta de los estudiantes}

1. ¿Me han resultado útiles las mini-lecciones en vídeo (screencast)?

- No las he visto / Nada útiles / Un poco útiles / Algo útiles / Muy útiles / Extremadamente útiles

2. En caso de NO haber visto los screencast, ¿cuáles son las razones que tienes para NO haberlos visto? (Indica todas las respuestas que se adapten a tu situación)

- No necesitaba ayuda adicional / Me olvidé de ver los screencasts / No tuve tiempo para ver los screencasts / Creo que los screencasts no son útiles / No pude encontrar los screencasts en PoliformaT / Tuve problemas técnicos con los screencasts / He utilizado otros recursos / Otros (especificar)

3. Indica los screencast que has visto (Selecciona de una lista).

4. Se pueden utilizar los screencasts de muchas maneras. ¿Cuál es la mejor descripción de

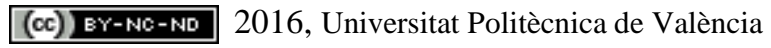

Congreso IN-RED (2016) 
cómo los has utilizado tú? (selecciona solamente una respuesta)

- Veo el vídeo completo de principio a fin / Veo grandes trozos en busca de información / Ojeo los screencast / Veo varias veces algunos segmentos / Voy a puntos específicos para revisarlos

5. De forma general, ¿cómo valoras los screencast?

- Muy malos / Malos / Regulares / Buenos / Muy buenos

6. Tengo una comprensión más profunda de algunos conceptos de la asignatura debido a los screencasts

- Totalmente en desacuerdo / En desacuerdo / Indeciso / De acuerdo / Totalmente de acuerdo

7. En general, ¿cuál era tú nivel de compresión de los conceptos antes de ver los screencast?

- Nula / poca / regular / buena / muy buena

¿Y después de ver los screencast?

- Nula / poca / regular / buena / muy buena

8. Prefiero repasar las clases (feedback) utilizando screencast, a revisar comentarios escritos por el profesor (apuntes, notas, ...)

- Totalmente en desacuerdo / En desacuerdo / Indeciso / De acuerdo / Totalmente de acuerdo

9. Prefiero repasar las clases utilizando screencast a revisar las transparencias de las clases

- Totalmente en desacuerdo / En desacuerdo / Indeciso / De acuerdo / Totalmente de acuerdo

10. La duración de los screencast me resulta, en general

- Muy corta / Corta / Adecuada / Larga / Muy larga

11. ¿Qué es lo que más te gusta de los screencast?/¿Qué es lo que menos te gusta de los screencast? (Pregunta abierta)

\subsection{Test de rendimiento académico}

La encuesta de satisfacción a los estudiantes se completó con una prueba en la que parcialmente se planteaban contenidos que se encontraban en los screencast. A partir de la misma se analizó si existía correlación entre la utilización de los screencast y la nota de la prueba. La nota global de la misma (en la que existían contenidos abordados por los screencast y otros que no lo eran) se utilizó para segmentar por nivel de conocimientos y establecer la valoración de los screencast según los mismos.

\section{Resultados}

\subsection{Encuesta de satisfacción}

De los 60 estudiantes que respondieron a la encuesta, 15 no vieron el screencast. Los resultados se presentan de acuerdo con los objetivos mencionados en la introducción.

A) ¿Los estudiantes incorporan screencast como parte de los materiales de apoyo al aprendizaje?

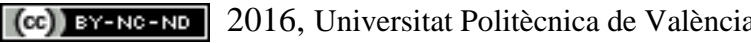

Congreso In-Red (2016) 


\section{P1. ¿Me han resultado útiles las mini-lecciones en vídeo (screencast)?}

El número de contestaciones y los porcentajes correspondientes a cada respuesta sobre el total alumnos que han visto los screencast se muestran en la Figura 1.

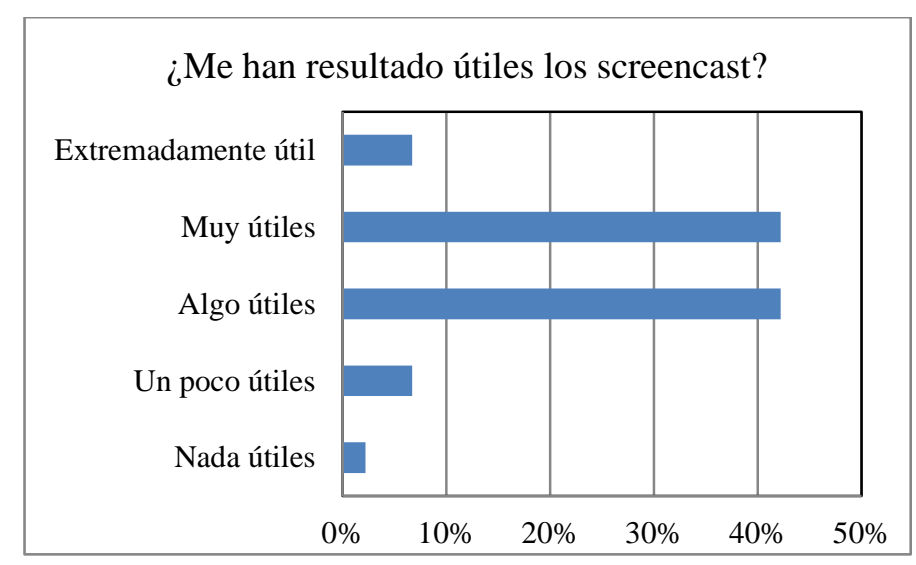

Fig. 1 Diagrama de barras correspondiente a la pregunta 1 de la encuesta.

Se puede observar que la mayoría de los estudiantes considera que las mini-lecciones en vídeo screencast han resultado desde algo útil a extremadamente útil. Menos del 10\% consideran que no era nada útiles o poco útiles. Si asignamos la puntuación de 1 cuando no les resulta nada útil, 2 si opinan que han sido un poco útiles, 3 si los encuentran algo útiles, 4 cuando los encuentran muy útiles y 5 en el caso de que los consideren extremadamente útiles, se obtiene una valoración promedio de 3,4 con una desviación estándar (SD) de 0,8, es decir, una valoración global positiva.

P2. En caso de NO haber visto los screencast, ¿cuáles son las razones que tienes para NO haberlos visto? (Indica todas las respuestas que se adapten a tu situación)

Tabla 2. Porcentaje de cada respuesta correspondiente a la pregunta 2 de la encuesta (los estudiantes pueden seleccionar más de una respuesta).

\begin{tabular}{|l|c|}
\hline \multicolumn{1}{|c|}{ Respuesta } & $\%$ \\
\hline No necesitaba ayuda adicional & 6,9 \\
\hline Me olvidé de ver los screencasts & 34,5 \\
\hline No tuve tiempo para ver los screencasts & 13,8 \\
\hline Creo que los screencasts no son útiles & 0 \\
\hline No pude encontrar los screencasts en PoliformaT & 6,9 \\
\hline Tuve problemas técnicos con los screencasts & 3,4 \\
\hline He utilizado otros recursos & 13,8 \\
\hline Otros (especificar) & 20,7 \\
\hline
\end{tabular}

Cuando se pregunta a los estudiantes acerca de las razones por las que no ven algunos de los screencast, se obtienen resultados muy variados. Es llamativo el alto porcentaje de estudiantes que olvidó verlos.

(cc) EY-NC-ND 2016, Universitat Politècnica de València 
P3. Marca el screencast que has visto (seleccionar de una lista).

Se ofreció a los estudiantes una lista de 7 screencast. Todos los screencasts habían sido vistos por varios estudiantes. La distribución de los screencast más buscados era más o menos uniforme a lo largo del tiempo.

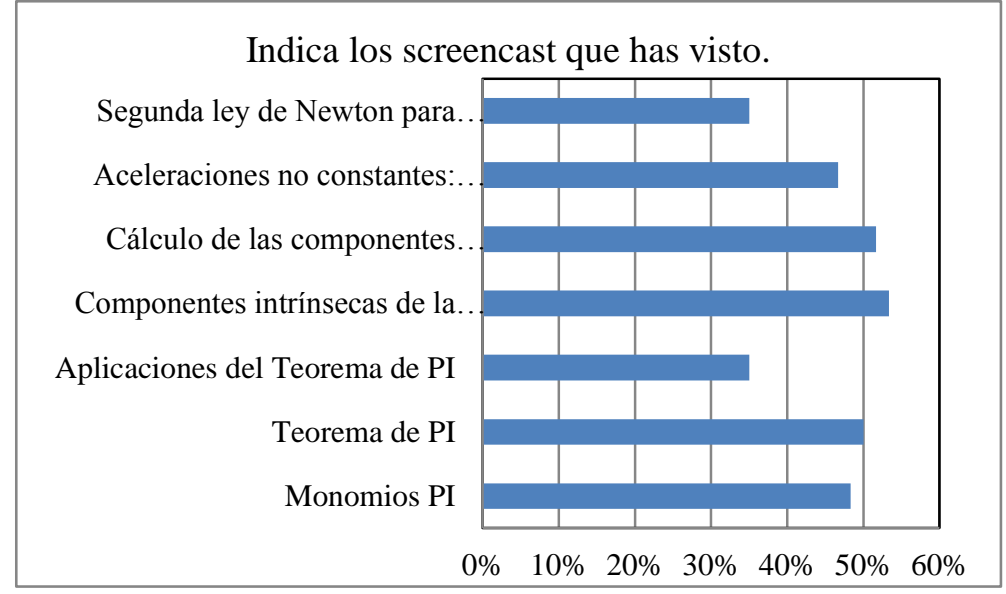

Fig. 2 Diagrama de barras correspondiente a la pregunta 3 de la encuesta.

P4. Se pueden utilizar los screencasts de muchas maneras. ¿Cuál es la mejor descripción de cómo los has utilizado tú? (selecciona solamente una respuesta)

Se pueden utilizar los screencasts de muchas maneras.

¿Cuál es la mejor descripción de cómo los has utilizado tú?

Voy a puntos específicos para revisarlos

Veo varias veces algunos segmentos

Ojeo los screencast

Veo grandes trozos en busca de información

Veo el vídeo completo de principio a fin

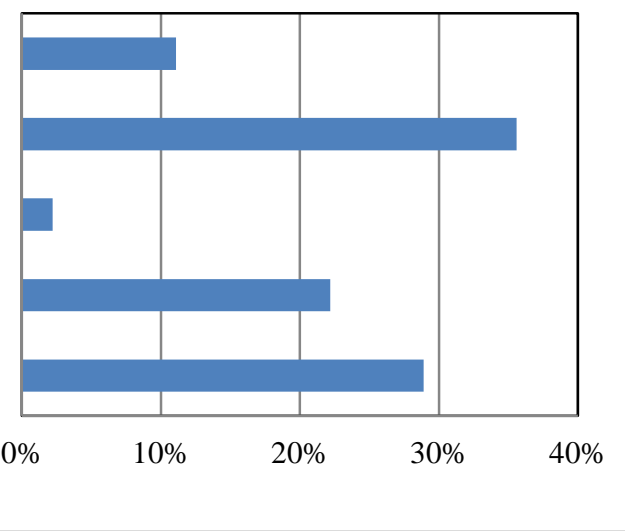

Fig. 3 Diagrama de barras correspondiente a la pregunta 4 de la encuesta.

En relación con la forma en que los estudiantes utilizan el screencast, el mayor porcentaje corresponde a encuestados que ven cierto segmento más de una vez, aunque algunos otros los miraban desde principio a fin y otros saltaron en busca de información específica.

P10. La duración de los screencast me resulta en general...

(cc) EY-NC-ND 2016, Universitat Politècnica de València

Congreso In-Red (2016) 


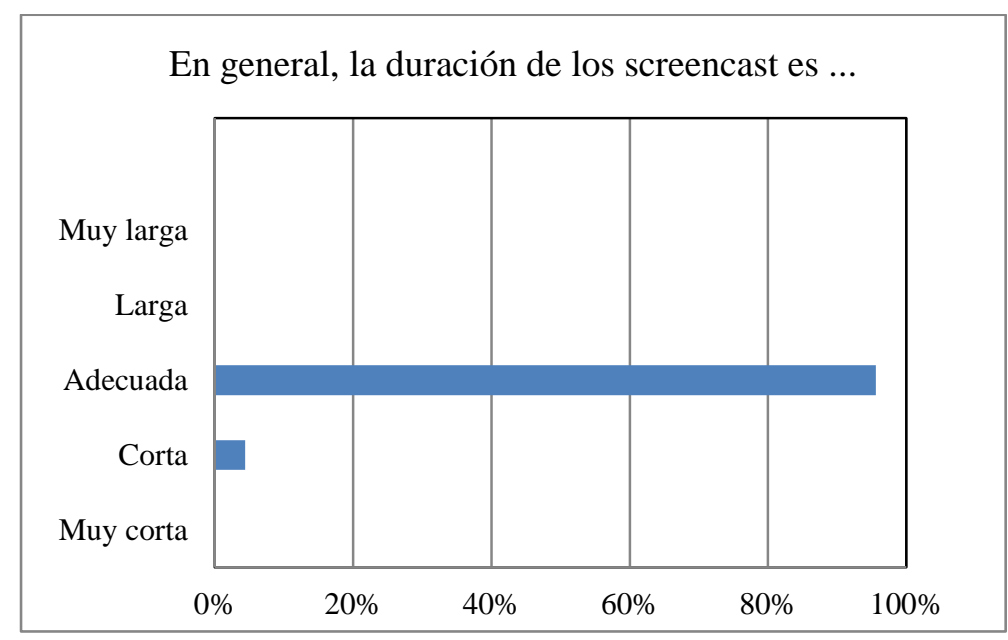

Fig. 4 Diagrama de barras correspondiente a la pregunta 10 de la encuesta.

La mayoría de los estudiantes piensan que la duración de los screencasts es la adecuada.

En resumen, los estudiantes consideran que el screencast es útil para su aprendizaje (puntuación 3,4 en la pregunta P1 de valoración). La razón para no ver screencast estaba más bien relacionada con el hecho de que los estudiantes aún no han incorporado esta herramienta en sus estudios de todos los días que a otras dificultades técnicas. Por otra parte, los estudiantes aprovechan la posibilidad que ofrece el screencast de verlo varias veces o de focalizarse en algunos aspectos (47\%), mientras que otros lo utiliza de una manera más tradicional, viéndolo entero o grandes trozos (51\%). Claramente, la longitud de los screencast se consideró adecuada.

\section{B) ¿Consideran los estudiantes que el screencast es útil en su estrategia de aprendizaje?}

P5. De forma general, ¿cómo valoras los screencast?

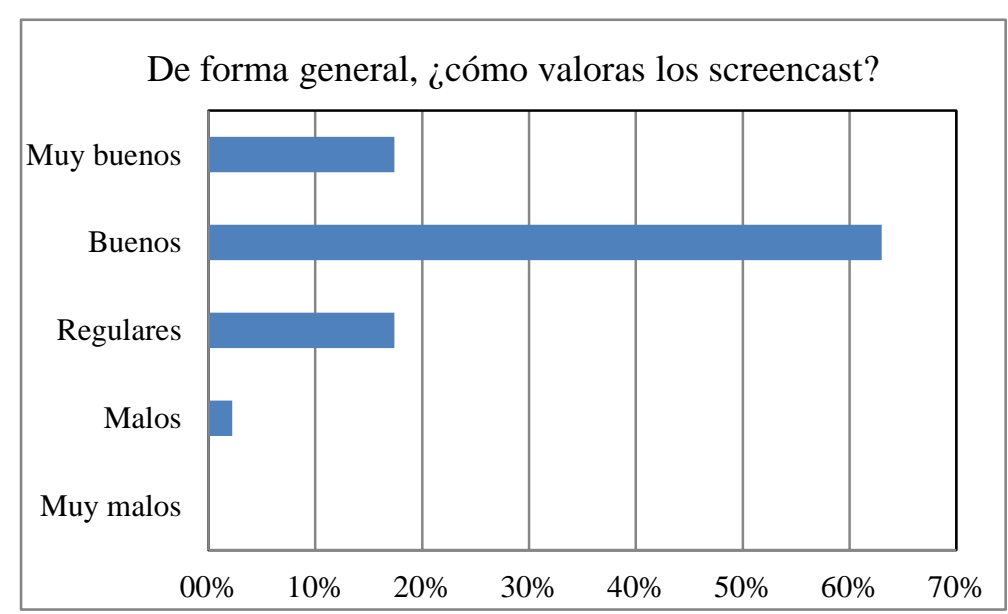

Fig. 5. Diagrama de barras correspondiente a la pregunta 5 de la encuesta. 
Casi todos los estudiantes consideran que la calidad del screencast aceptable, buena o muy buena. El $80 \%$ considera que es buena o muy buena. Asignando a cada respuesta la misma puntuación comentada anteriormente (de 1 a 5) se obtiene una valoración global de 4,0 con una desviación estándar (SD) de 0,7.

P6. Tengo una comprensión más profunda de algunos conceptos de la asignatura debido a los screencasts

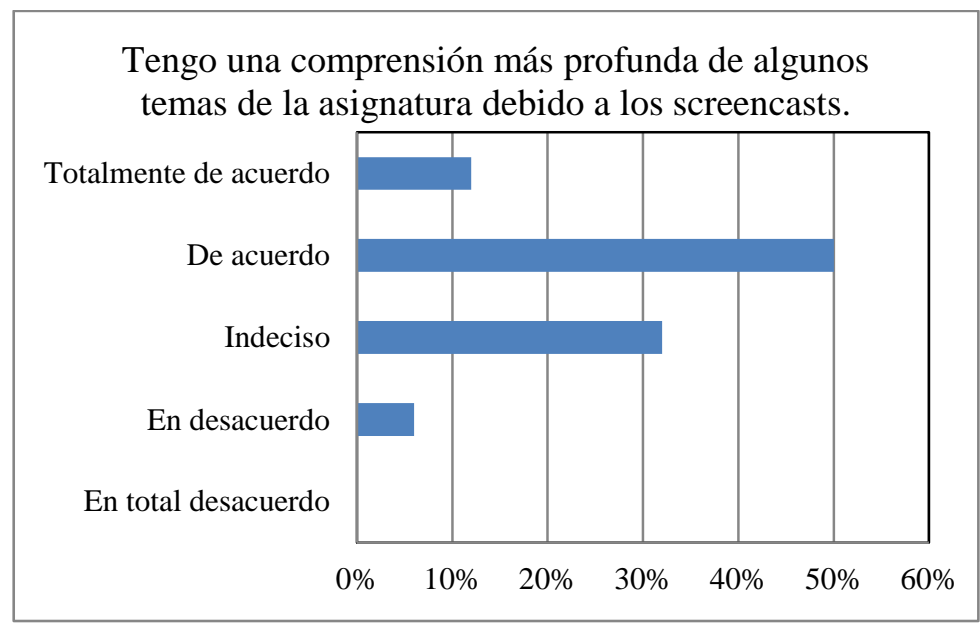

Fig. 6 Diagrama de barras correspondiente a la pregunta 6 de la encuesta.

Más de la mitad de los estudiantes (52\%) consideran que obtienen una comprensión más profunda al ver el screencast y el $27 \%$ no se deciden al respecto. La valoración media que se obtiene, aplicando la puntuación a cada respuesta es $3,7(\mathrm{SD}=0,8)$.

P7. En general, ¿cuál era tú nivel de compresión de los conceptos antes de ver los screencast? ¿Y después de ver los screencast?

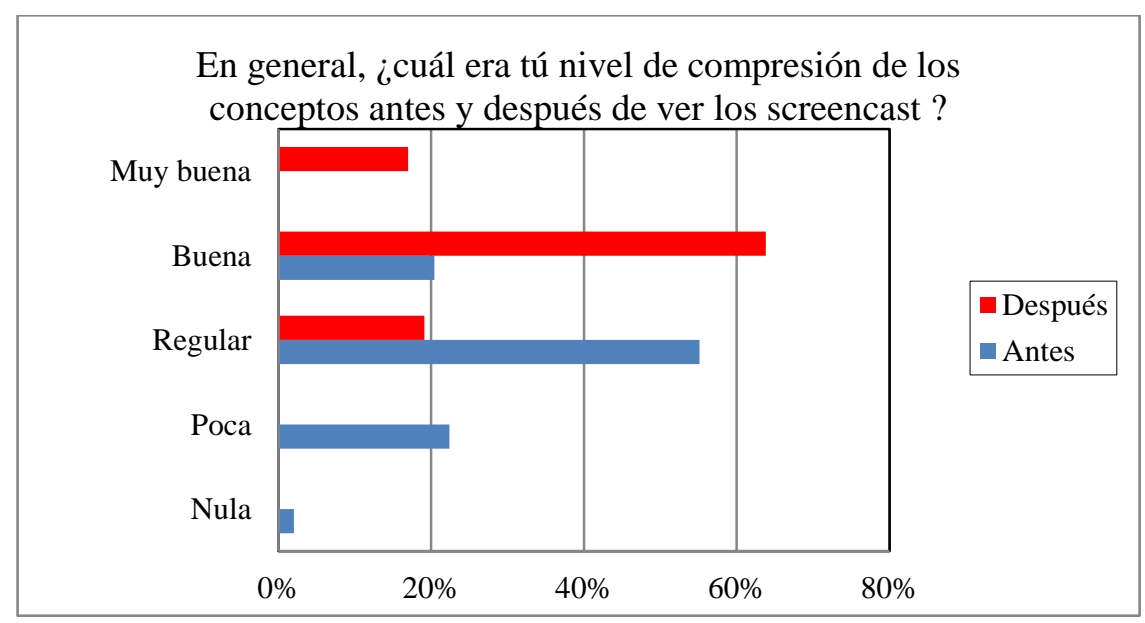

Fig. 7 Diagrama de barras correspondiente a la pregunta 7 de la encuesta.

Cuando se compara el nivel de comprensión antes y después de ver el screencast, se puede observar claramente que aumenta después de ver el screencast. Mientras que el $24 \%$ de los 
estudiantes tienen ningún o algún nivel de entendimiento antes de ver el screencast, ninguno de ellos se mantuvo en el mismo nivel después de ver el screencast. Y además ningún estudiante tenía muy buen nivel antes de ver el screencast mientras que el $17 \%$ tenían muy buen nivel de comprensión después de verlos. Si se asigna la puntuación de 1 a 5 se puede cuantificar de forma global la mejora percibida por los estudiantes como consecuencia de utilizar los screencast. Ésta mejora es clara, ya que pasa de una puntuación global de 3,1 $(\mathrm{SD}=0,8)$ a 4,0 $(\mathrm{SD}=0,6)$ después de utilizar losa screencast.

\section{C) ¿Prefieren screencast en lugar de otros materiales de apoyo?}

P8. Prefiero repasar las clases (feedback) utilizando screencast, a revisar comentarios escritos por el profesor (apuntes, notas, ...)

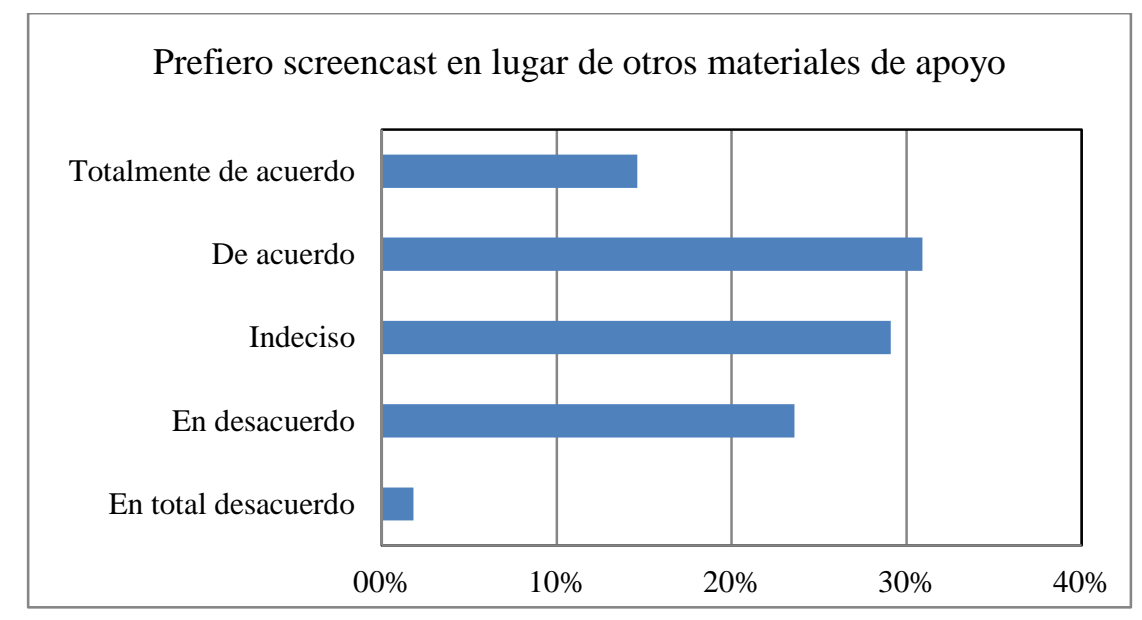

Fig. 8 Diagrama de barras correspondiente a la pregunta 8 de la encuesta.

Aproximadamente un $50 \%$ de los alumnos $(45,4 \%)$ están totalmente de acuerdo o de acuerdo con la afirmación, mientras que un $25 \%$ están total o parcialmente en desacuerdo con la misma, siendo el número de indiferentes elevado (29\%). La valoración global que se obtiene es de 3,3 $(\mathrm{SD}=1,0)$.

P9. Prefiero repasar las clases utilizando screencast a revisar las transparencias de las clases 


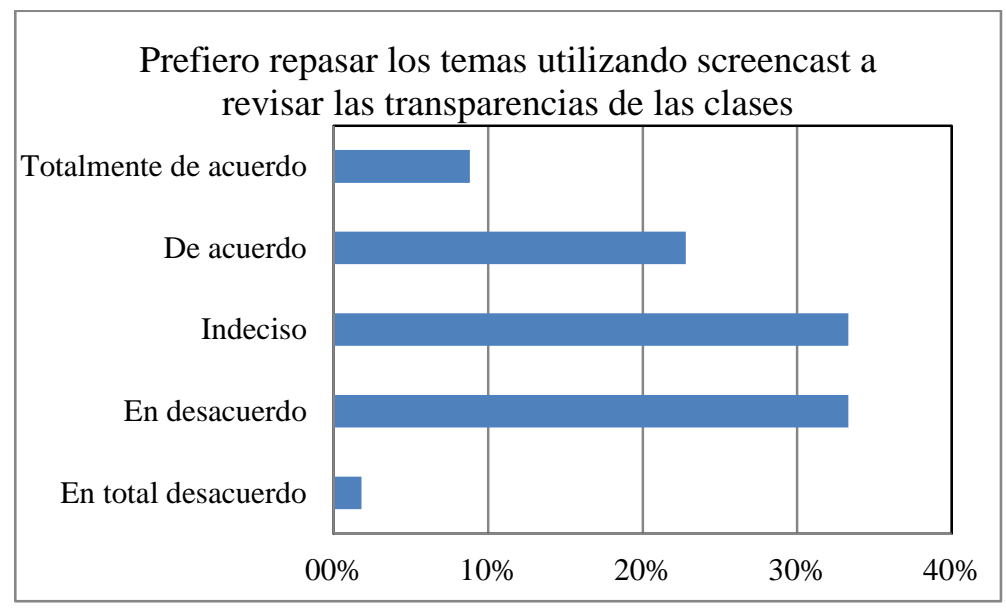

Fig. 9 Diagrama de barras correspondiente a la pregunta 9 de la encuesta.

En este caso un 35\% se decanta por preferir las transparencias mientras que un 32\% por los screencast, siendo el 33\% indiferente. En este caso se obtiene una valoración global de 3,0 $(\mathrm{SD}=1,0)$.

\subsection{Test de rendimiento académico}

Con el fin de obtener una visión más clara de los resultados obtenidos, y dar respuesta a las preguntas de investigación planteadas en la introducción, "¿Mejoran los screencast el rendimiento académico de los alumnos?”, se ha realizado un análisis estadístico de los resultados obtenidos en una prueba de contenidos.

\subsubsection{Efecto de los Screencast sobre el rendimiento}

Se realiza un estudio de las medias de los grupos (grupo G0: no han visto los vídeos / grupo G1: los han visto) para ver si hay diferencias significativas entre ellos. Se analiza una cuestión del examen (puntuación 1 sobre 10) y un problema (puntuación 3 sobre 10) que tienen una relación directa con los contenidos mostrados en los screencast. La suma de las dos notas está normalizada sobre 10 .

Como resultado se encuentra que el grupo G0 (alumnos que no han visto el screencast) tiene nota superior al G1 (alumnos que sí han visto el screencast) $(4,9 / 4,7)$ ), sin embargo no hay diferencias significativas $(t=-0,38 ; p=0,71)$. Seguidamente comparamos la nota media de examen de cada grupo, ya que el nivel de conocimientos de los alumnos puede introducir un sesgo en el resultado. Como resultado se encuentra que el grupo que no ha visto los screencast (G0) tiene nota superior al grupo de estudiantes que sí ha visto los screencast (G1) $(5,8 / 4,9)$, no encontrándose diferencias significativas $(t=-1,37 ; p=0,18)$.

Los resultados de la primera comparación quedan explicados por la segunda. Aunque no se encuentran diferencias significativas, la tendencia hacia una mayor nota del grupo de los que no han visto los vídeos, queda ampliamente justificada por su mayor nota global en el examen. 
A la vista de estos resultados, se hace necesario experimentos con mayor potencia estadística para valorar el efecto de los screencast en el rendimiento académico de los estudiantes.

\subsubsection{Valoración de los vídeos según estratos de rendimiento}

Como ya se ha comentado al analizar la pregunta número 5 de la encuesta, los alumnos asignan a los screencast una valoración global de 4,0. Con objeto de analizar el nivel de aceptación por cualificación de los estudiantes, se analizan estos resultados por grupos obtenidos al segmentar por terciles relativos a la nota del examen. En el grupo 1 se sitúan los estudiantes con una nota inferior a 4,1; en el grupo 2 los estudiantes con una nota comprendida entre 4,1 y 5,8; y en el grupo 3 los que han obtenido una nota superior a 5,8. Entre estos tres grupos, se obtiene que los alumnos con calificación media y superior valoran mejor los screencast que los de calificación menor (figura 10): G2=4,2 (desviación estándar 0,7); G3=4,1 (desviación estándar 0,5); G1= 3,6 (desviación estándar 0,2); encontrándose además diferencias significativas al realizar un Test Anova $(F=4,88$; $p<0,05)$.

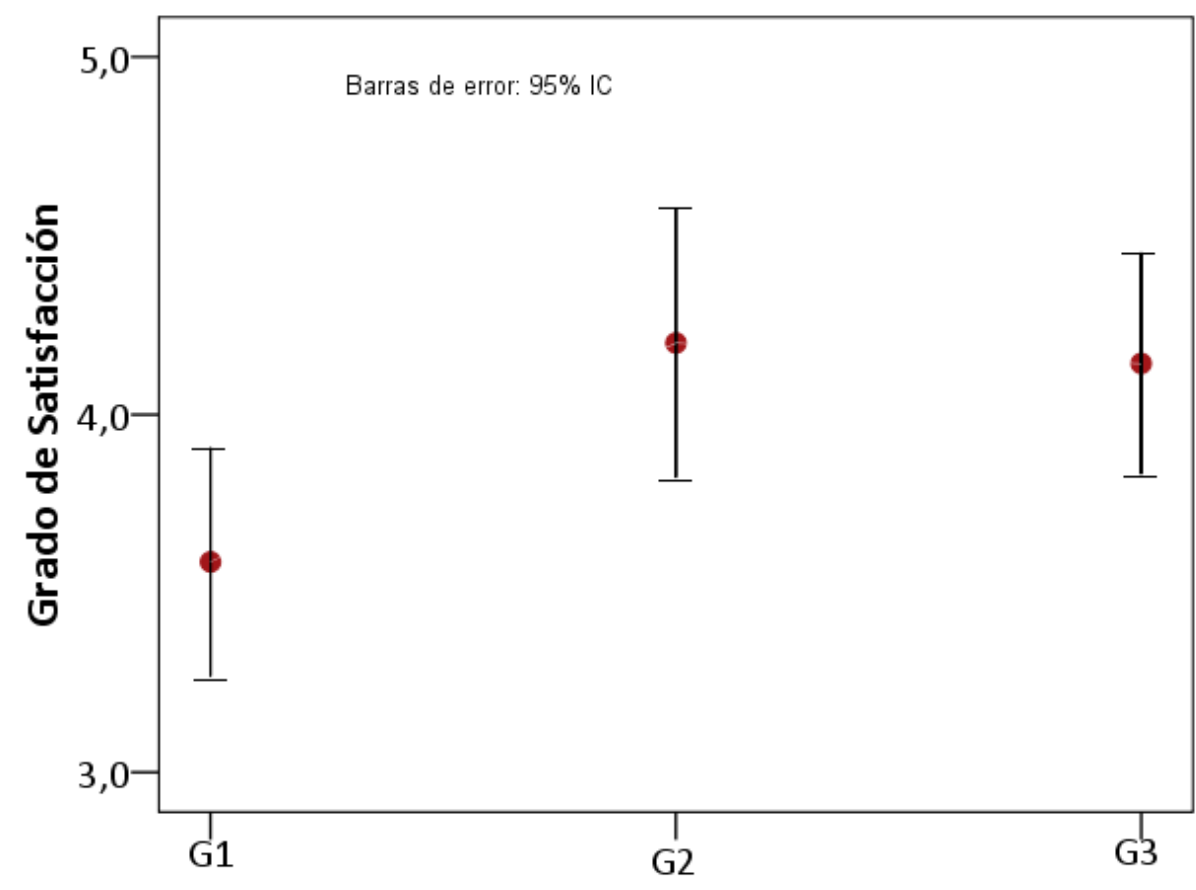

Grupos

Fig. 10 Grado de satisfacción por grupos obtenidos al segmentar por terciles relativos a la nota del examen. G1: estudiantes con una nota inferior a 4,1; G2: estudiantes con una nota comprendida entre 4,1 y 5,8; G3: estudiantes que han obtenido una nota superior a 5,8. 
Con objeto de encontrar los grupos entre los que se encuentran estas diferencias, se realiza un contraste "post hoc", utilizando el método DMS (Diferencia Mínima Significativa). Los resultados del mismo se muestran en la tabla 3.

Tabla 3. Comparaciones múltiples entre los tres grupos en los que se han dividido los alumnos según la nota obtenida en el examen. G1: nota<4,1; G2: 4,1 $\leq$ nota $\leq 5,8$; G3: nota $\geq 5,8$.

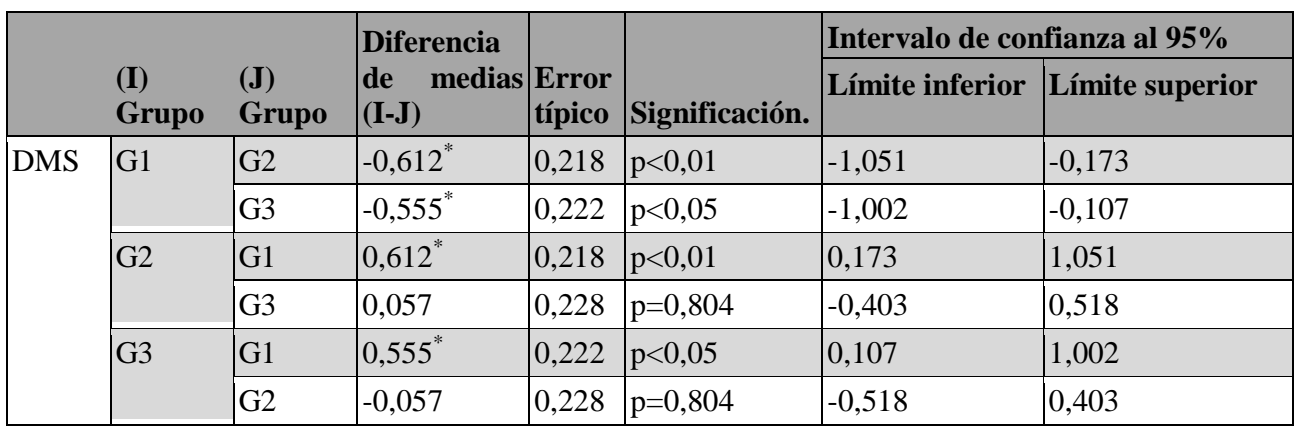

* La diferencia de medias es significativa al nivel 0.05 .

Del análisis se deduce que el G1 muestra diferencias significativas con el G2 ( $<<0,01)$ y con el G3 ( $\mathrm{p}<0,05)$, no encontrándose diferencias significativas entre el G2 y el G3 $(\mathrm{p}=0,804)$.

El resultado obtenido muestra que aunque los alumnos valoran positivamente los vídeos, aquellos pertenecientes al tercil de calificación inferior valoran menos positivamente los mismos, siendo las diferencias significativas frente a los del tercil medio y alto.

\subsection{Pregunta de respuesta abierta}

En la pregunta abierta de la encuesta, se pidió a los estudiantes opinión sobre lo que les gustaba y lo que no le gustaba de los screencast. En esta sección, las fortalezas y debilidades que encuentran se pusieron de relieve, junto con las propuestas que hicieron. En el texto que sigue, las declaraciones del estudiante de la pregunta abierta se identifican por comillas.

En general, consideraron que "las grabaciones de pantalla proporcionan un refuerzo para el estudio" y dijeron que "puedo comprobar los vídeos y tomar notas como en clase". Hicieron hincapié en la claridad y precisión de las explicaciones, que es una explicación guiada de los conceptos más complejos paso a paso y más fácilmente comprensible. Hicieron hincapié en la ventaja de tener audio junto con las diapositivas y los screencasts proporcionaron la seguridad para revisar los contenidos de la materia (dijeron que a veces sus notas de clase eran incorrectas o incompletas).

Algunos de ellos lo consideraron similar a una clase cara a cara "se puede ver el desarrollo, los comentarios del profesor y las aclaraciones, de manera similar a una clase cara a cara", aunque otros lo destacaban frente a la clase cara a cara, porque "la explicación de algunos conceptos es mucho mejor" y "puedo aumentar mi compresión de las áreas no explicadas

(c)) EY-NC-ND 2016, Universitat Politècnica de València

Congreso In-Red (2016) 
en las diapositivas". Es interesante que lo consideraban como una herramienta adicional, útil para el aprendizaje: "Los videos no son la única o la mejor herramienta para el estudio, sino que complementan a otras".

Generalmente utilizaron los screencasts para repasar, "ayudan a resolver dudas y profundizar", "los veo antes de hacer el examen", "puedo comprobar si he utilizado la metodología correcta", "que ayudan a estudiar y revisar conceptos que no tenía claros", "puedo ir a los detalles que yo era incapaz de ver en clase y repasar algunas demostraciones",...

Muchos estudiantes destacaron varias ventajas de los screencast: siempre accesible, se puede ver tantas veces como se quiera, parando y volviendo, etc. "Siempre tienes a tu disposición la explicación del profesor en cualquier momento", "tantas veces como se desee", "la clase cara a cara no se puede repetir o detener".

Algunos estudiantes cuestionaron el contenido de los videos porque "no reflejan la totalidad del procedimiento de resolución" o por la falta de generalidad, "muy específico". Sin embargo, tienen una valoración positiva porque exigen más contenido: "no hay grabaciones de pantalla de cada parte del temario", "se debería aplicar a más ejercicios".

Los estudiantes también señalaron la limitación de la enseñanza a distancia: la ausencia del profesor. No hay una respuesta inmediata a sus preguntas, "no es posible hacer preguntas al screencast", "en el aprendizaje es importante transmitir las dudas al profesor".

Los estudiantes querían un video más dinámico: en algunos casos "se limita a leer lo que está escrito en las diapositivas", "se requiere una explicación más dinámica", "el ritmo me parece lento".

Indicaron problemas técnicos con la captura de audio y la imagen de los videos ("el audio no era bueno", "el volumen es muy bajo", "el maestro cubre algunos datos"), y la plataforma de Internet que aloja los vídeos ("toma algún tiempo saltar dentro del video", "a veces la imagen parpadea"), que es un problema frecuente en aplicaciones que dependen de la calidad de las conexiones a Internet.

Los estudiantes han identificado las posibilidades de las grabaciones de pantalla en su proceso de aprendizaje. Ellos hicieron propuestas que parecían indicar que aceptarían video-notas y docencia inversa: vídeos "al igual que hemos hecho en clase, incluyendo preguntas de los estudiantes", "video-notas en lugar de archivos PDF y tener que asistir a clases", ¿qué pasaría si se nos permitiera estudiar la teoría en casa y las horas de clase se usaran para la resolución de problemas?" en resumen, se cuestionaban "un modelo de educación en el que tenemos que asistir a clases".

\section{Conclusiones}

Los estudiantes incorporan los screencast como parte del material de apoyo para el aprendizaje, aunque no han incorporado totalmente esta herramienta a su rutina de trabajo. Por ello, la mitad de ellos aun lo utilizan siguiendo métodos tradicionales (lo ven de

(cc)) EY-NC-ND 2016, Universitat Politècnica de València 
principio a fin) frente a la otra mitad que utiliza sus características especiales (revisión de un fragmento varias veces). Los estudiantes consideran útil al screencast en su tarea de aprendizaje, siendo conscientes de las mejoras y ayuda a la profundización de su aprendizaje que aporta. Aunque los screencasts se han propuesto como material complementario, no como una alternativa de otros materiales educativos como libros, apuntes del profesor o diapositivas, cuando se les ha pedido a los estudiantes que los comparen no muestran una respuesta clara. Como tendencia podría indicarse que prefieren las diapositivas del profesor más que los screencasts y estos más que los apuntes del profesor.

Cuando fueron invitados a expresar sus opiniones a través de una pregunta abierta destacaron varias ventajas de los screencast: siempre accesible, se puede ver tantas veces como se quiera, parando y volviendo, etc. aunque también señalaron las principales ventajas del aprendizaje cara a cara, la posibilidad de hacer preguntas.

Al correlacionar la encuesta de satisfacción con el nivel de conocimiento de los alumnos, evidenciado a través de una prueba de conocimiento, se ha puesto de manifiesto que los alumnos de nivel superior o medio valoran los screencast más que aquellos de nivel inferior, lo que constituye un resultado relevante. Por otro lado no se han obtenido evidencias significativas sobre el uso de los screencast y el mayor o menor rendimiento académico. Por ello se considera relevante continuar la investigación en esta línea, a través de pruebas de alta potencia estadística.

Mientras tanto, a partir de los resultados obtenidos se puede concluir, que los estudiantes están bien preparados para un futuro con una educación mixta, donde las nuevas tecnologías serán una parte no separable de los materiales escritos tradicionales.

\section{Agradecimientos}

Los autores desean agradecer al Instituto de Ciencias de la Educación de la Universitat Politècnica de València (España) el apoyo al Grupo de Innovación Docente e-MACAFI y el apoyo financiero a través del proyecto PIME/2014/A025.

\section{Referencias}

BRAUN, V. y CLARKE, V., 2006. Using thematic analysis in psychology. Qualitative Research in Psychology, vol. 3, no. 2, pp. 77-101. DOI 10.1191/1478088706qp063oa.

DUNN, P.K., MCDONALD, C. y LOCH, B., 2015. StatsCasts: screencasts for complementing lectures in statistics classes. International Journal of Mathematical Education in Science and Technology, vol. 46, no. 4, pp. 521-532. DOI 10.1080/0020739X.2014.990530.

GREEN, K.R., PINDER-GROVER, T. y MILLUNCHICK, J.M., 2012. Impact of Screencast Technology: Connecting the Perception of Usefulness and the Reality of Performance. Journal of Engineering Education, vol. 101, no. 4, pp. 717-737. DOI

(cc)) BY-NC-ND 2016, Universitat Politècnica de València

Congreso In-Red (2016) 
10.1002/j.2168-9830.2012.tb01126.x.

JORDAN, C., LOCH, B., LOWE, T., MESTEL, B. y WILKINS, C., 2012. Do short screencasts improve student learning of mathematics? MSOR Connections. DOI 10.11120/msor.2012.12010011.

LIKERT, R., 1932. A technique for the measurement of attitudes. Archives of Psychology, vol. 22, pp. 1-55.

MAYER, R.E., 2014. The Cambridge Handbook of Multimedia Learning (2nd Edition). New York: Cambridge University Press.

MCDONALD, C., DUNN, P.K., LOCH, B. y WEISS, V., 2013. StatsCasts: supporting student learning of introductory statistics. Proceedings of the 9th Delta Conference of Teaching and Learning of Undergraduate Mathematics and Statistics 2013, pp. 2429.

MERKT, M., WEIGAND, S., HEIER, A. y SCHWAN, S., 2011. Learning with videos vs. learning with print: The role of interactive features. Learning and Instruction. DOI 10.1016/j.learninstruc.2011.03.004.

OUD, J., 2009. Guidelines for effective online instruction using multimedia screencasts. Reference Services Review, vol. 37, no. 2, pp. 164-177. DOI 10.1108/00907320910957206.

PETERSON, E., 2007. Incorporating screencasts in online teaching. International Review of Research in Open and Distance Learning, vol. 8, no. 3.

SUGAR, W., BROWN, A. y LUTERBACH, K., 2010. Examining the anatomy of a screencast: Uncovering common elements and instructional strategies. International Review of Research in Open and Distance Learning, vol. 11, no. 3, pp. 1-20.

SUTTON-BRADY, C., SCOTT, K.M., TAYLOR, L., CARABETTA, G. y CLARK, S., 2011. The value of using short-format podcasts to enhance learning and teaching. Research in Learning Technology, vol. 17, no. 3, pp. 219-232. DOI 10.3402/rlt.v17i3.10878.

TUNKU AHMAD, T.B. y DOHENY, F., 2014. Six key benefits of screencasts in learning Maths: an Irish case study. 24 junio 2014. S.1.: CRC Press.

VINCELETTE, E.J. y BOSTIC, T., 2013. Show and tell: Student and instructor perceptions of screencast assessment. Assessing Writing, vol. 18, no. 4, pp. 257-277. DOI 10.1016/j.asw.2013.08.001. 